\title{
RESEARCH ON VISUAL ANALYSIS METHODS OF TERRORISM EVENTS
}

\author{
Wenyue Guo ${ }^{\mathrm{a}, \mathrm{b}}$, Haiyan Liu ${ }^{\mathrm{a},{ }^{*}}$, Anzhu $\mathrm{Yu}^{\mathrm{a}, \mathrm{b}}, \mathrm{Jing} \mathrm{Li}^{\text {a }}$ \\ ${ }^{a}$ Institute of Surveying and Mapping, Zhengzhou, China. \\ ${ }^{\mathrm{b}}$ State Key Laboratory of Geo-Information Engineering, Xi'an, China.
}

Commission II, WG II/3

KEY WORDS: Terrorism Event, Visual Analysis, Spatio-temporal Thematic Map, Statistical Charts, GTD

\begin{abstract}
:
Under the situation that terrorism events occur more and more frequency throughout the world, improving the response capability of social security incidents has become an important aspect to test governments govern ability. Visual analysis has become an important method of event analysing for its advantage of intuitive and effective. To analyse events' spatio-temporal distribution characteristics, correlations among event items and the development trend, terrorism event's spatio-temporal characteristics are discussed. Suitable event data table structure based on " $5 \mathrm{~W}$ " theory is designed. Then, six types of visual analysis are purposed, and how to use thematic map and statistical charts to realize visual analysis on terrorism events is studied. Finally, experiments have been carried out by using the data provided by Global Terrorism Database, and the results of experiments proves the availability of the methods.
\end{abstract}

\section{INTRODUCTION}

According to statistical data from Global Terrorism Database (GTD), worldwide terrorism events has begun to show the trend of more and more frequent since 2004 , and the number of occurrences each year increases rapidly. Improving the analytical ability of responding to terrorism events has become an important aspect of governments govern ability. At present, some international community has specialized agencies and researchers studying on terrorism event data organization and analysis. Such as, the Global Terrorism Database (GTD, 2015) and the Rand Corporation (Rand, 2015), these institutions not only have large-scale event database of normal structure and relatively complete content, but analysis spatio-temporal distribution characteristics of terrorism events and terrorism organizations as well, their statistic charts and analysis reports are shared publicly. Utilization of data mining (Qiliang, 2011, Guangqiang, 2010) and knowledge discovery, statistical analysis (Jiadong, 2011) and visual analysis technology (Jianzhong, 2012, Yu, 2008) to analyse and predict events has becoming a very urgent and meaningful work. With the advantage of intuitive, visual analysis is one of the main methods of event analysis, it can effectively reduce the cost of information analysis by turning data into graph or chart (Thomas, 2005, Wong, 2004).

Visual analysis uses statistical thematic maps and charts to convert data into graphs or diagrams to show the characteristics of data intuitively. Many scholars have researched the methods of producing statistical thematic maps and graphs and the mechanism of event visual analysis. A visual method by using parallel coordinates, bar charts and area charts to display the uncertainty of measure results has been proposed (Huaiqing, 2012). Li (Daichao, 2014) has researched the methods visual analysis of crime big data from the view of representation content, representation methods and interaction design.

The existing visual analysis researches on event-oriented data mostly focused on the designs and producing methods of statistical symbol, statistical thematic maps or interaction system. There is no discussion of the intrinsic and the spatio- temporal characteristics of terrorism events, and also lacking of researches on the applicability of different visual analysis methods and their application in auxiliary decision support analysis. This paper uses the data from Global Terrorism Database (GTD), discussed the spatio-temporal characteristics of terrorism events, designed the structure of event data table that based on $5 \mathrm{~W}$ theory, and then researched and analysed the application category of different kinds of thematic maps and statistical charts which are event-oriented. Through thematic maps and statistical charts analysis the characteristics of spatiotemporal distribution range, the associations between property items and the overall trend of terrorism events. Then, researched and analysed the applicable area of different types of thematic maps and thematic diagrams for terrorism event visual analysis.

\section{EVENT CHARACTERISTICS AND DATA PROCESSING}

\subsection{Characteristics of Terrorism Events}

Individual uncertainty and the overall trend. Terrorism events are affected coupling by political, economic, religious, demographic, geographical and many other factors, the uncertainty in spatio-temporal distribution makes it difficult to forecast for a single specific event. But through the research on lots of and a wide range of events data, it can be found that events with same attribute term appear to have some laws in spatio-temporal distribution and overall trend.

Relevance concealment. Terrorism events always occur suddenly, but there is still correlation between event internal attributes item, such as, event time, event location, the manipulating organization and target type. But all these relationships are too difficult to be found directly from events data.

\subsection{Event Data Table Structure Design}

Completed and standard event database is the basic requirement for visual analysis. There is no uniform international standard

\footnotetext{
${ }^{*}$ Corresponding author
} 
for terrorism event data structure, each database is different in event data structure. GTD uses dozens of property items including event type, event embodiments and weapon types to describe the event characteristics completely. It is quite complex and highly disruptive to describe the relationships between dozens of dimensions. Rand database put amounts of information into "Description" attribute by long text. Neither data provided by GTD nor the data provided by the Rand database are directly suitable for visual analysis, since there are redundant data, incomplete data nor duplicate data. Thus, source data need to be cleaned up and integrated. Also, suitable data table structure has to be designed.

\begin{tabular}{|lllllllll|}
\hline eventid & iyear & imonth & iday & $\ldots$ & country & country_txt & region & region_txt \\
\hline 200601000008 & 2006 & 1 & 0 & $\ldots$ & 4 & Afghanistan & 6 & South Asia \\
200601020002 & 2006 & 1 & 2 & $\ldots$ & 95 & Iraq & 10 & Middle East\&North Africa \\
200601020003 & 2006 & 1 & 2 & $\ldots$ & 186 & Sri Lanka & 6 & Middle East\&North Africa \\
\hline
\end{tabular}

Table 1 Event data provided by GTD

\begin{tabular}{|llllllll|}
\hline Date & City & Country & Perpetrator & Weapon & Injuries & Fatalities & Descriprion \\
\hline 2-Jan-06 & Baghdad & Iraq & Unknown & Explosive & 0 & 0 & Usama al-Najafai, the... \\
2-Jan-06 & Baghdad & Iraq & Unknown & Firearms & 3 & 2 & Gunmen opened fire on a ... \\
2-Jan-06 & Yairipok Malom & India & PULF & Firearms & 0 & 1 & In Thoubai district, a leader... \\
\hline
\end{tabular}

Table 2 Event data provided by Rand

According to the $5 \mathrm{~W}$ propagation mode proposed by Losswell (who says what in which channel to whom with what effects), five universal event properties are extracted: temporal attribute (when), spatial properties (where), event embodiment (who), event type (what) and event target (whom). Terrorism event is a kind of social phenomena with temporal characteristics, spatial location characteristics. Complete event data table structure that can reflect event's spatio-temporal characteristics and convenience for stastic should include the time item, spatial location item and universal thematic property feature items. The event structure table is shown in Table 3.
Time item: time record of event, it may be time, or may be time period.

Spatial location item: record of event location, depending on the accuracy of the data source, location information may be accurate to "province", "city" or a specific latitude and longitude coordinates.

Thematic property feature item: the property items always depends on the user needs, but the universal thematic property should include unique event code, event type, the performing organization and the objective type.

\begin{tabular}{|c|c|c|c|c|c|c|}
\hline Time & Country & Province & Latitude & Longitude & Attack type & Target type \\
\hline $2007 / 1 / 19$ & Iraq & Baghdad & 33.296 & 44.342 & Armed Assault & Private Citizens \& Property \\
\hline $2007 / 3 / 1$ & Iraq & Diyala & 33.75 & 44.633 & Kidnapping & Police \\
\hline $2014 / 12 / 28$ & Syria & Aleppo & 34.888 & 38.351 & Bombing/Explosion & Terrorists/Non-State Militia \\
\hline $2014 / 12 / 29$ & Syria & Homs & 34.6 & 37.083 & Bombing/Explosion & Military \\
\hline
\end{tabular}

Table 3 Event data table structure

\section{EVENT VISUALIZATION AND VISUAL ANALYSIS}

Spatio-temporal thematic map and statistical charts are the main methods for terrorism events visual analysis (Daichao, 2014). Spatio-temporal thematic maps focus on the performance of the spatial and temporal distribution of events and the development of state, can be applied into trend analysis, high-threat area analysis and auxiliary planning for arresting route. Statistical charts focus on showing the relationships as contrast, association and variation of event property items by using of statistical data, in order to analyse the internal mechanisms of events and the active pattern of terrorism organizations.

\subsection{Spatial Temporal Thematic Map}

3.1.1 Event Visualization: Since different accuracy of data sources, event's geographical information may be accurate to "country", "province", "city" or latitude and longitude. Depending on the scope of the event, it can be represented as a point, linear or planar area on the map. Distribution of global terrorism events that happened in the year of 2014 is just as

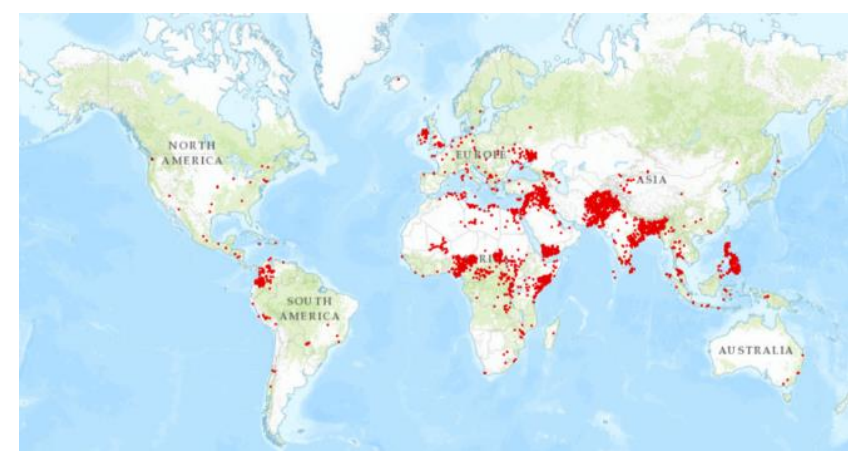

Figure 1 Distribution of global terrorism events of year 2014

Adding time element on spatial thematic map can help to perform the development of terrorism events' spatial and thematic characteristics in time domain. Time factor can be expressed in two ways: horizontal time axis and vertical time layers. 


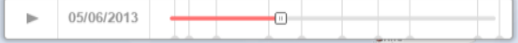

Figure 2 Horizontal time axis

Horizontal time axis: Each specific coordinate point on the timeline corresponds to a spatial distribution of terrorism events. Through continuously varying the time coordinates of the point to make events' spatial expression exhibited dynamic and trend, as shown in Figure 3 provided by GTD. This varying way over time that time elements and spatial elements linked in focused on the performance of regulars of how spatial elements change over time variation.

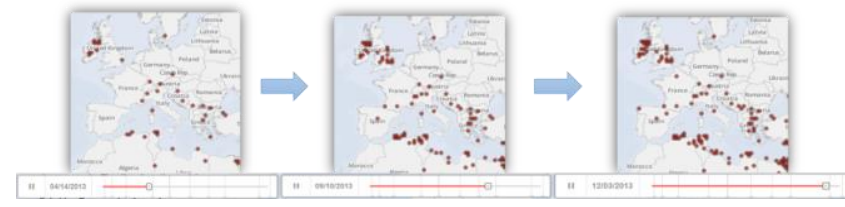

Figure 3 Dynamic event presentation map by using horizontal time axis

Vertical time layers: The whole time domain is divided into several time interval, and according to the time item, event data are storage in different layers, different time intervals corresponding to different event display layers, as shown in Figure 4. Event layer changes within a specific time zone, not continuous display. This time element expression focused on performing the differences of events' spatial distribution and attribute elements in different time interval.

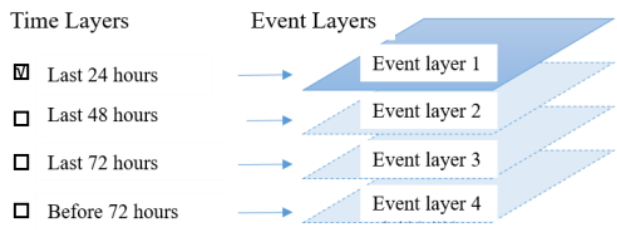

Figure 4 Correspondence between vertical time layers and event display layers

3.1.2 Event Analysis Based on Thematic Map: Thematic map can show the spatio-temporal distribution characteristics of events. Through further analyse by using thematic maps, potential distribution of hot spots and evolution of events can be discovered.

Trend analysis: Through spatio-temporal thematic map, events' development can be well represented. Spatio-temporal thematic map is used for the analysis how spatial distribution of events changing over time.

Hot spot analysis: Hot spot analysis refers to regional hot spot analysis. When the event is difficult to find the difference of frequency distribution because large amount of points coordinate is overlapped. Through calibration of different areas in different colours according to the number of events, the discrepancy of events frequency distribution can be performed. As the difference between Figure 1 and Figure 5, users can directly find that Iraq, Syria and Afghanistan are the most frequency areas that terrorism events occur.

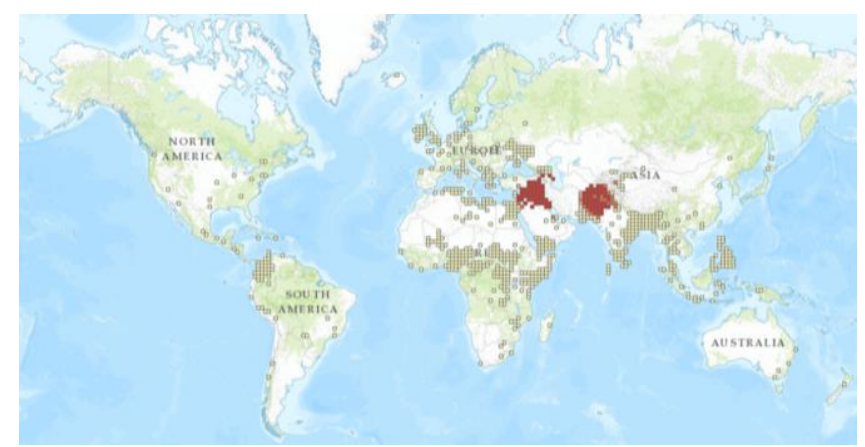

Figure 5 Hot spot analysis of worldwide terrorism events in 2014

Buffer analysis: Buffer analysis is mainly used in designated escape routes and avoid danger, and also for the rapid determination of the scope of the arrest. When terrorism event occurs, civilians often require rapid escape from the danger zone, and avoid entering other threatened areas. Criminals fled rang is a buffer area around event point, in this way buffer analysis can help quickly determine the raids range.

\subsection{Event Statistical Charts}

Statistical chart is an important visual analysis means, and traditional statistical charts including bar charts, line charts, pie charts, etc. These kinds of charts are mainly used for show the condition, constitutes of phenomenon and entity, or the relationships among them (Wattenberg, 2005). With the development of computer visualization technology and information technology, the type and performance capability of statistical charts have greatly enriched. According to the content that chart performs, common charts are divided into three categories:

Trend charts: trend analysis charts are based on Cartesian coordinate system, including line charts, bar charts, scatter charts, funnel charts, event river charts, etc. Trend analysis charts record the values of statistical indicators by the horizontal axis coordinate scale. The up and down of lines, changes of the columns' width and height or saturation, and the dimensions of scatterplots are used to measure distinguishes among statistical indicators. When the horizontal axis represents time, the statistical chart is mainly used to analysis and find out the statistic indicator's hot time spots and how it changes over time. When the horizontal axis represents categories, the statistical chart is always used to analysis the distinguishes between multiple statistical indicators, the most common one of this type is bar chart, and the bar chart can be converted to radar chart when expressed in polar coordinate system. Bar chart and line chart are often used in combination to show both the category differences and the trend, the composite chart is shown in Figure 6. In spatial coordinate system, through superimposing column statistical symbols, point symbols and planar symbols onto basemap, statistical thematic map can be formed which could reflect both the value of statistical indicators and the characteristics of spatial distribution, as shown in Figure 7. 


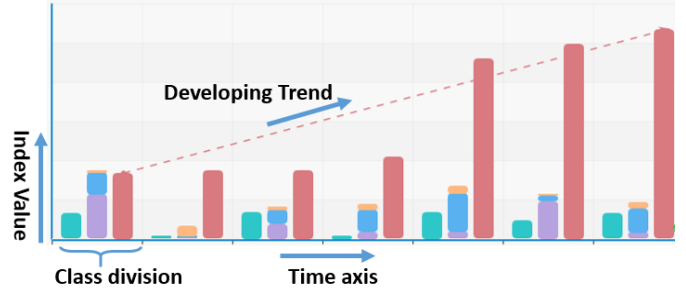

Figure 6 A trend analysis composite chart based on Cartesian coordinates

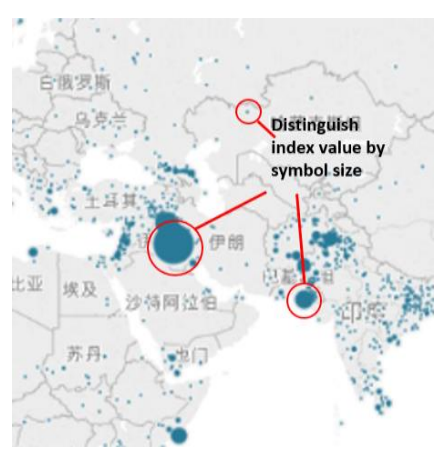

Figure 7 Superimpose statistical symbol on the map

Proportion charts: including pie charts, donut charts, rose charts, radar charts, rectangular tree charts, etc. Proportion analysis charts are based on Polar coordinate system, including pie charts, donut charts, rose charts and so on. This king of chart is focused on analysing the components and component composition of event's property. Pie charts, donut charts and rose charts focus on analysing the contrast between the constituent components. Central angle is used to record the size of each component, the length of radius or the area of circle or sector is used to record the statistic value of each component, with different colours to distinguish between the various components. Proportion analysis charts mainly used for threaten region and target analysis.

Relation charts: including chord charts, force charts, tree map, Gantt charts, etc. (Yubin, 2008). The relationships between terrorism events includes direct internal associations and external indirect associations. Direct internal association refers to the relationships between event's $5 \mathrm{~W}$ attributes. The statistical indicators are expressed as ring or circular symbols, the relationship between various indicators are expressed as lines, and the width of line or the amount of lines are used to measure the correlation degree between indicators. Relation analysis charts are mainly used to analysis the target type of terrorism organization and the organization's active patterns. Direct internal relation analysis charts include chord charts, force charts, tree maps and so on. Indirect external association refers to the order of events in temporal space, cycle or clustering features which are non-causal associations.

Six visual analysis types are put forward about terrorism event, the data object, explanation and suitable chart types are listed in Table 4

Charts focus on reflecting statistical characteristics, and without having spatial intuitive. Sometimes, through overlapping column, line or composite structure statistical map symbols on basemap, both spatial distribution of event and statistical characteristics can be reflected. As shown in Figure 8, pie charts are overlapped on the world map, and through this method, we could see that Iraq has been one of the world' s most frequency countries from 2006 to 2013.

\begin{tabular}{|c|c|c|c|}
\hline $\begin{array}{c}\text { Visual } \\
\text { analysis }\end{array}$ & Data objects & Explanation & $\begin{array}{c}\text { Usage } \\
\text { statistic chart } \\
\text { type }\end{array}$ \\
\hline $\begin{array}{l}\text { Hot spot } \\
\text { analysis }\end{array}$ & $\begin{array}{l}\text { Event } \\
\text { frequency }\end{array}$ & $\begin{array}{l}\text { Reflecting the } \\
\text { hot spatial area } \\
\text { or hotspot time } \\
\text { interval }\end{array}$ & $\begin{array}{l}\text { Line chart, bar } \\
\text { chart, scatter } \\
\text { chart }\end{array}$ \\
\hline $\begin{array}{l}\text { Threat } \\
\text { analysis }\end{array}$ & $\begin{array}{l}\text { Statistics of } \\
\text { different } \\
\text { kinds of } \\
\text { events or } \\
\text { events } \\
\text { carried out } \\
\text { by different } \\
\text { organizations }\end{array}$ & $\begin{array}{l}\text { Reflecting } \\
\text { main types of } \\
\text { threats or } \\
\text { major threaten } \\
\text { organization }\end{array}$ & $\begin{array}{l}\text { Pie chart, } \\
\text { donut chart, } \\
\text { radar chart, } \\
\text { rose chart, } \\
\text { rectangular } \\
\text { tree chart }\end{array}$ \\
\hline $\begin{array}{l}\text { Vulnerable } \\
\text { target analysis }\end{array}$ & $\begin{array}{l}\text { Attacked } \\
\text { frequency } \\
\text { statistics of } \\
\text { different type } \\
\text { target }\end{array}$ & $\begin{array}{l}\text { Reflecting } \\
\text { vulnerable } \\
\text { targets and } \\
\text { type of threat } \\
\text { within spatio- } \\
\text { temporal area }\end{array}$ & $\begin{array}{l}\text { Pie chart, } \\
\text { donut chart, } \\
\text { radar chart, } \\
\text { rose chart, } \\
\text { rectangular } \\
\text { tree chart }\end{array}$ \\
\hline Trend analysis & $\begin{array}{l}\text { Event } \\
\text { frequency in } \\
\text { continuous } \\
\text { time interval }\end{array}$ & $\begin{array}{l}\text { Reflecting } \\
\text { events or event } \\
\text { attribute } \\
\text { changes over } \\
\text { time }\end{array}$ & $\begin{array}{l}\text { Line chart, bar } \\
\text { chart, event } \\
\text { river chart }\end{array}$ \\
\hline $\begin{array}{l}\text { Internal } \\
\text { correlation } \\
\text { analysis }\end{array}$ & $\begin{array}{l}\text { Correlations } \\
\text { among event } \\
\text { thematic } \\
\text { feature items }\end{array}$ & $\begin{array}{l}\text { Reflecting } \\
\text { relationships } \\
\text { of internal } \\
\text { thematic items } \\
\text { as } \\
\text { embodiment, } \\
\text { event type and } \\
\text { target type }\end{array}$ & $\begin{array}{l}\text { chord chart, } \\
\text { force chart }\end{array}$ \\
\hline $\begin{array}{l}\text { External } \\
\text { Relational } \\
\text { analysis }\end{array}$ & $\begin{array}{l}\text { Event spatio- } \\
\text { temporal } \\
\text { feature items }\end{array}$ & $\begin{array}{l}\text { Reflecting the } \\
\text { relationship } \\
\text { between } \\
\text { events }\end{array}$ & Gantt chart \\
\hline
\end{tabular}

Table 4 Event visual analysis types and suitable statistic charts

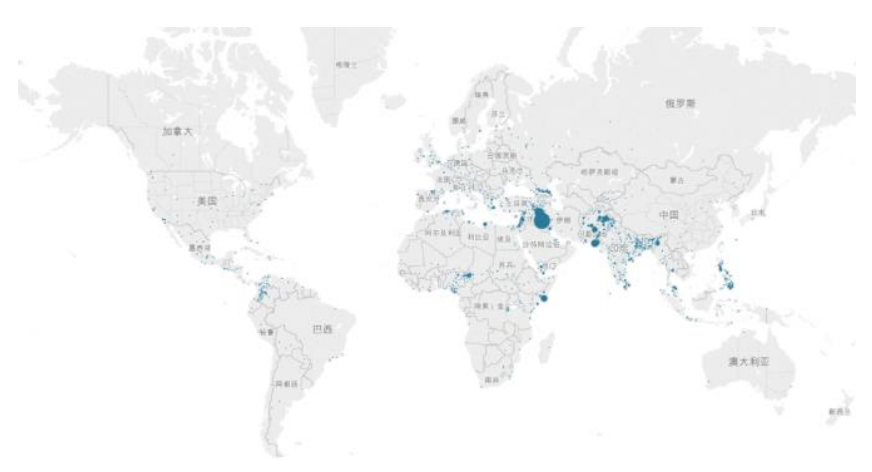

Figure 8 Pie chart of terrorism events that happened throughout the world from 2006 to 2013

\section{EXPERIMENT AND ANALYSIS}

According to 1753 terrorism events that carried out by ISIS organization from 2007 to 2014 recorded by GTD. Using visual analysis methods based on ArcGIS Pro and ECharts 2.7 (http://echarts.baidu.com) to analysis the spatio-temporal active area, key target type and distribution of different attack means of ISIS. 


\subsection{Experiment Procedure}

Firstly, design an event data table structure, including time item, spatial item as latitude, longitude, belonging country and city, event embodiment, event type and target type and so on.

Then, clean up and integration the source data, form a complete specification of event data set.

Locate the events, and then analyse the active range of ISIS. It can be seen from Figure 9 that from 2007 to 2009, ISIS mainly active in Iraq, but since 2010, this organization began to spread to Syria, Turkey and Egypt.

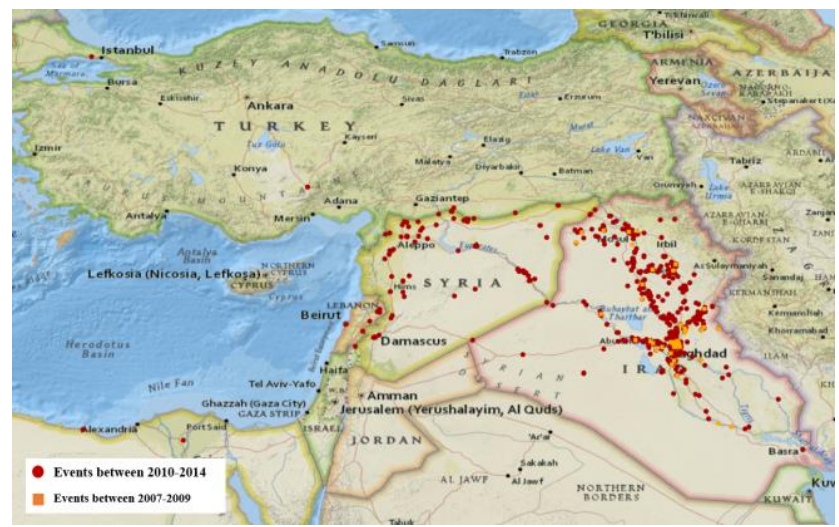

Figure 9 Active range of ISIS from 2007 to 2014

According to statistic, use bar chart to analysis the temporal active range of ISIS, use radar chart and rose chart to analysis the main target types and main means of attack, then chord chart is used to analysis the relationship between attack means and target types. According to the data provided by GTD, ISIS has carried out armed attacks for 164 times, kidnapping for 196 times, bombings for 1143 times, assassination for 58 times, facilities destroy 28 times, barricade incident 8 times, hijacking 4 times and a few terrorism events of fuzzy types.

Temporal active range analysis: Monthly mean frequency is the radio of events number in each month and the number of events throughout the year. Through mean frequency, the organization's temporal active range can be well analysed. As can be known according to Figure 10, ISIS active most frequent in July in the year of 2013 while active most in September in 2014. In general, ISIS active more from July to September, so measures should be strengthened especially during this period of time.

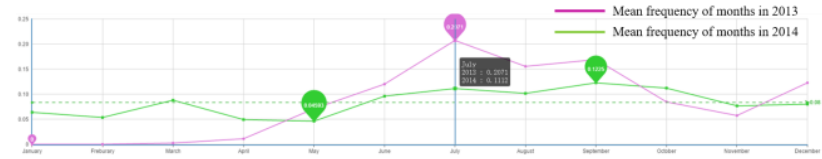

Figure 10 Mean frequency of ISIS from January to December in 2013 and 2014

Threaten temporal analysis: During different time, ISIS focus on different types of target. As Figure 11 shows, during the January, ISIS mainly focus on private citizens, police and military. During the May, the organization focus on private citizens, police and religious institutes. According this radar chart, conservation goals should always change depending on the time.

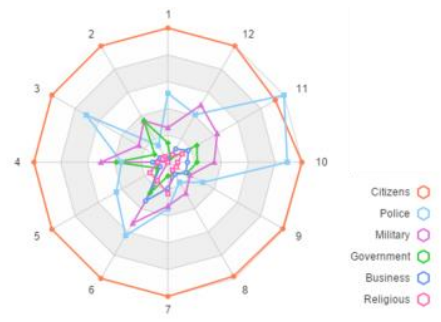

Figure 11 Event targets in different months

Threaten type analysis: Different targets always face with different kinds of threat type. Rose chart is used to show the type and extent of threat some target face. As is shown in Figure 12 , bombing is in the highest level of threat to private citizens and police, but to journalist and media, kidnapping is the most serious threat. So, citizens and police should strengthen preventive measures against bombing attacks, while media institute should strengthen prevention against kidnapping.

Analysis of the relationship between event type and target type: According to statistics, chord chart is drawn to show the relationships between event types and target types. As is shown in Figure 13, different kinds of events always focus on different kinds of targets, the main targets of kidnapping private citizens, military and police while the main targets of assassination events are military, police and government. By using chord chart, users can directly see if there are association between some event type and target type, and how much is the associate degrees.

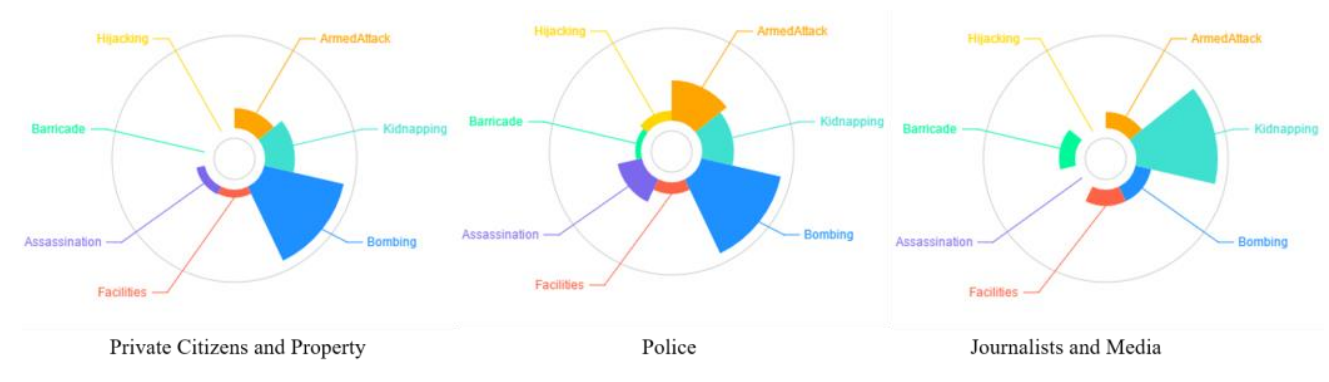

Figure 12 Type and extent of threat of different targets 


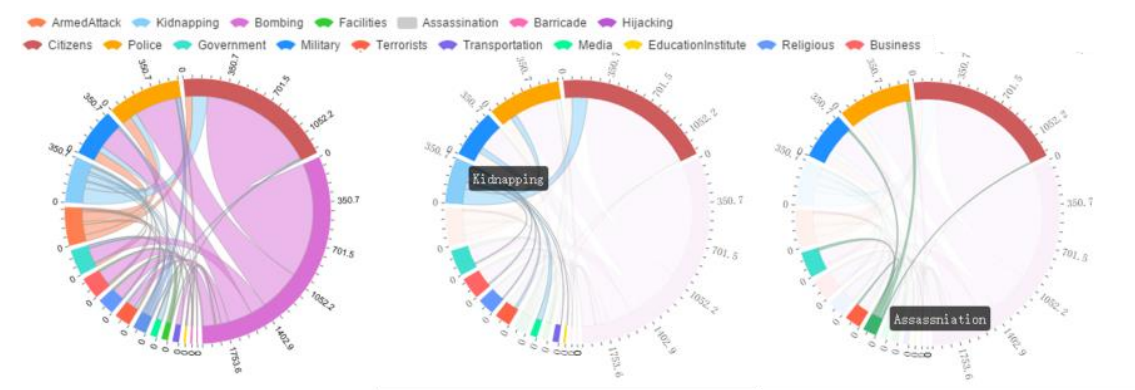

Figure 13 Relationships between event types and target types

\section{SUMMARY}

This paper mainly discuss the visual analysis methods applied to terrorism events, analysis spatio-temporal characteristics of terrorism events and design event data structure for visual analysis. Put forward the method to analysis the spatio-temporal distribution, correlation relationships and trend of terrorism events by using thematic map and spatio-temporal statistical charts. Sum up six class event analysis types and discuss suitable visual analysis methods to each of them. Finally, verify the feasibility of the method by experiments. Through experiments, it has been found that ISIS organization actives most frequent from June to October during a year. The organization actives mainly in Iraq and Syria, and in recent years, it began to spread to other countries. The main target is civilian, police and military. It still has following problems:

Using statistical charts for different types of analysis, but not form an event analysis system. Integration is the next step that will be studied.

The reliability of visual analysis results mainly based on statistical data, how to verify the correctness of analysis results integrated with data mining method also requires further study.

This paper splits event's multidimensional properties into multiple two dimensional properties, visual analysis method for high integrated and uniformity multidimensional event data needs to be further researched.

\section{ACKNOWLEDGEMENTS}

This work is funded by State Key Laboratory of Geoinformation Engineering (NO. SKLGIE2015-M-3-1) and the National Natural Science Foundation of China (Nos. 41471387, 415014446).

\section{REFERENCES}

Global Terrorism Database, 2015.http://www.start.umd.edu/gtd/.

Rand Database of Worldwide terrorism incidents, 2015. http://www.rand.org/.

Qiliang L., Min D., Yan S., 2011. A novel spatial clustering method based on multi-constraints. Acta Geodaetica et Cartographica Sinica, 40(4), pp. 509-516.

Guangqiang L., Min D., Weiling Z., 2010. Events-coverage based spatio-temporal association rules mining method. Journal of Remote Sensing, 14(3), pp. 468-481.

Jiadong Z. The main characteristics and development trend of World Terrorism. International Review, (5), pp. 8-15.
Jianzhong, G., Yi C., Wenqi F., 2010. Research and implementation on the visualization of network public opinion of emergence. Surveying and Mapping of Geology and Mineral Resources, 28(4), pp. 5-8.

Thomas J. J., Cook K. A.,2005. Illuminating the path: the research and development agenda for visual analytics. Computer Graphics.

Wong P. C., Thomas J. 2004. Visual analytics. In: IEEE Transactions on Visualization and Computer Graphics Computer Graphics and Applications,24(5), pp. 20-21.

Huaiqing $\mathrm{H}, \mathrm{He} \mathrm{W}, 2012$. Uncertainty measurement and visual analysis on terroristic attacks data. Computer Engeering and Science, 34(9) pp: 77-82.

Daichao L., Sheng W., 2014. Theme-oriented visual analysis of crime with big data. Journal of Geo-information Science. 16(5), pp. 735-745.

Wattenberg M., 2005. Baby names, visualization, and social data analysis. In: Proceeding of IEEE Symposium on Information Visualization.

Yubin Y., Ning L., Yao Z., 2008. Networked data mining based on social network visualizations. Journal of Software, 19(8), pp. 1980-1994.

Revised April 2016 